\title{
Mechanism of the increased ventilatory response to exercise in patients with chronic heart failure
}

\author{
N P Buller, P A Poole-Wilson
}

\begin{abstract}
Minute ventilation, respiratory rate, and metabolic gas exchange were measured continuously during maximal symptom limited treadmill exercise in 30 patients with stable chronic heart failure. The ventilatory response to exercise was assessed by calculation of the slope of the relation between minute ventilation and rate of carbon dioxide production. There was a close correlation between the severity of heart failure, determined as the maximal rate of oxygen consumption, and the ventilatory response to exercise. Reanalysis of the data after correction for ventilation of anatomical dead space did not significantly weaken the correlation but reduced the slope of the relation by approximately one third. These results show that the increased ventilatory response to exercise in patients with chronic heart failure is largely caused by mechanisms other than increased ventilation of anatomical dead space. This finding supports the concept that a significant pulmonary ventilation/ perfusion mismatch develops in patients with chronic heart failure and suggests that the magnitude of this abnormality is directly related to the severity of chronic heart failure.
\end{abstract}

Patients with chronic heart failure show an increased ventilatory response to exercise. ${ }^{1-3}$ The size of this abnormality is related to the severity of chronic heart failure. ${ }^{34}$ It has also been established that patients with uncomplicated chronic heart failure maintain essentially normal arterial blood gas concentrations, both at rest and during exercise..$^{5-7}$ Consequently the increased ventilatory response that accompanies exercise is hyperpnoea and not hyperventilation, because hyperventilation, by definition, would cause a fall in the arterial concentration of carbon dioxide.

The mechanisms responsible for exercise hyperpnoea in patients with chronic heart failure are poorly understood. Commonly the hyperpnoea is attributed to an increased ventilation of anatomical dead space secondary to an altered relation between tidal volume and respiratory rate. ${ }^{3}$ This greater dependence on increasing respiratory rate rather than on tidal volume during exercise in patients with chronic heart failure is claimed to minimise respiratory work in the presence of "stiff" lungs. Recently, it has been suggested that an additional cause of hyperpnoea is an increase in physiological dead space $^{5-8}$ secondary to a ventilation/perfusion mismatch. ${ }^{8}$ The aims of the present study were, first, to determine the relative contributions of these two mechanisms to the measured ventilatory response during exercise in patients with chronic heart failure; and, second, to relate the measured ventilatory response during exercise to the maximum rate of oxygen consumption where the maximum rate of oxygen consumption was regarded as an indicator of the severity of heart failure.

\section{Patients and methods}

We studied 30 patients (mean age 53 years (range 30-69); 28 men) with stable chronic heart failure. Heart failure was caused by ischaemic heart disease in 19 , by dilated cardiomyopathy in eight, and followed replacement of the aortic valve in three. All patients had been in heart failure for more than 6 months. No patient had had an acute coronary event in the preceding three months. All patients were in sinus rhythm. All were being treated with diuretics and none was receiving $\beta$ antagonists. No patient had signs or symptoms suggestive of primary lung disease; formal respiratory function tests were not performed.

Exercise testing was performed according to the standard Bruce protocol with the addition of a stage $0(1 \mathrm{mph}(1.6 \mathrm{~km} / \mathrm{h})$ at $5 \%$ gradient $)$. The exercise laboratory was air conditioned with room temperature maintained in the range $20-21^{\circ} \mathrm{C}$. All patients were familiar with treadmill exercise and measurement of respiratory gas exchange. Patients continued their normal medication on the day of exercise testing, and all tests were performed at least three hours after meals.

Minute ventilation, the rate of oxygen consumption, and the rate of carbon dioxide production were measured continuously by the method of Davies and Denison. ${ }^{9}$ Validation and reproducibility of this method in our laboratory have been detailed in an earlier paper. ${ }^{10}$ In brief, patients were required to breathe through a two way respiratory valve (Collins) held by an adjustable head support. The expiratory port was connected via flexible tubing to a 71 mixing box (Airspec). Argon was added to the inlet port of the mixing box at a constant flow rate. After full mixing the resultant gas mixture was continuously analysed by a mass spectrometer (Airspec 20MGE). The analogue output from the mass spectrometer was fed to the analogue to digital
Lung Institute, SW3 6LY.

Accepted for publication 9 January 1990
The National Heart National Heart N P Buller

Correspondence to Professor P A Poole-Wilson, 
input channels of a microcomputer (BBC model B). Analogue to digital conversions were performed every $200 \mathrm{~ms}$ for each of the four channels (nitrogen, oxygen, carbon dioxide, and argon). Every $30 \mathrm{~s}$ a mean value for each channel was calculated, and from these mean values minute ventilation, the rate of oxygen consumption, and the rate of carbon dioxide production were derived. Values were corrected for atmospheric pressure, laboratory temperature, water vapour pressure, and body weight. We calculated the rate of oxygen consumption in $\mathrm{ml} / \mathrm{min} / \mathrm{kg}$ at standard temperature and pressure (dry) (STPD), the rate of carbon dioxide production in $\mathrm{ml} / \mathrm{min} / \mathrm{kg}$ STPD, and minute ventilation as $1 / \mathrm{min}$ at body temperature, pressure, and saturation (BTPS).

The respiratory rate was determined from a continuous recording of the temperature variation at the mouth obtained from a thermistor mounted inside the respiratory valve. Computation of minute ventilation was corrected for the dead space of the two way respiratory valve $(98 \mathrm{ml})$.

Anatomical dead space was calculated for each patient using the formula:

Anatomical dead space $=$ weight $\times 2 \cdot 2+$ age where weight and age are measured in kilograms and years respectively. ${ }^{11}$

Patients were encouraged to exercise until symptoms were intolerable. After each exercise test the ventilatory response to exercise was calculated. The total ventilatory response was determined as the slope of the relation between minute ventilation and the rate of carbon dioxide production. The alveolar ventilatory response was determined as the slope of the relation between alveolar ventilation (minute ventilation less the product of anatomical dead space and respiratory rate) and the rate of carbon dioxide production. Both values were obtained by the method of least squares linear regression.

The maximal rate of oxygen consumption $\left(\mathrm{VO}_{2} \max \right)$ was defined as the rate of oxygen consumption at peak exercise.

\section{Results}

All exercise tests were performed without complication. The limiting symptom in all patients was breathlessness. $\dot{\mathrm{VO}}_{2} \max$ ranged from $8 \cdot 7$ $\mathrm{mg} / \mathrm{kg} / \mathrm{min}$ to $19 \cdot 1 \mathrm{ml} / \mathrm{kg} / \mathrm{min}$ indicating moderate to severe heart failure. The relation between minute ventilation and the rate of carbon dioxide production was linear in all patients by visual inspection and the correlation coefficient was $r=0.98(0.04)$ mean $(S D)$. Figure 1 shows the raw data obtained from three patients. The last few points in the patient with the steepest slope in figure 1 do deviate from a linear relation. There is scatter of the last points in the other two patients illustrated. These small deviations from linearity do not materially affect the correlation coefficient or the calculation of the slope. The slope of the relation between minute ventilation and the rate of carbon dioxide production (total ventilatory response) was related to the severity of chronic heart failure. Figure 2 shows the relation between $\dot{\mathrm{V}}_{2} \max$ and total ventilatory response for all patients $(r=0.86$, slope $=-0.32, \mathrm{p}<0.001)$. Figure 3 shows the relation between $\dot{\mathrm{V}}_{2}$ max and alveolar ventilatory response $(\mathrm{r}=0 \cdot 85$, slope $=-0 \cdot 21$, $\mathrm{p}<0.001)$.

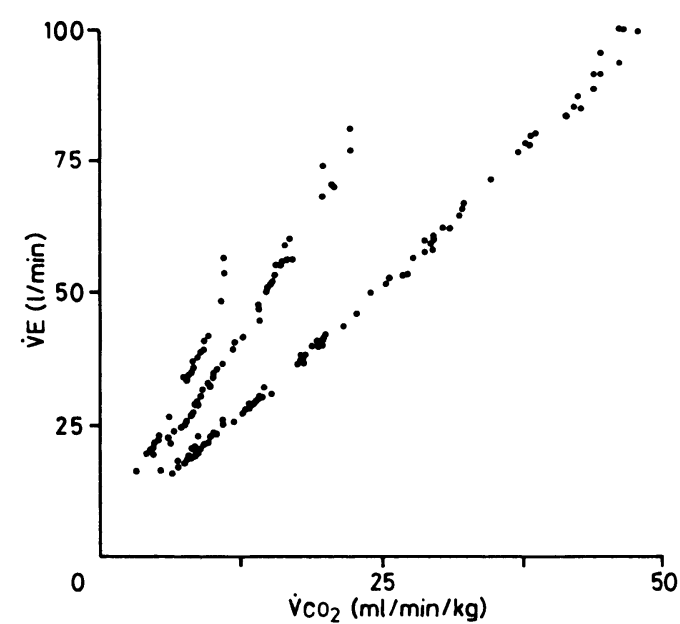

Figure 1 Example of the relation between minute ventilation and the rate of carbon dioxide production for three patients with chronic heart failure of differing severity.

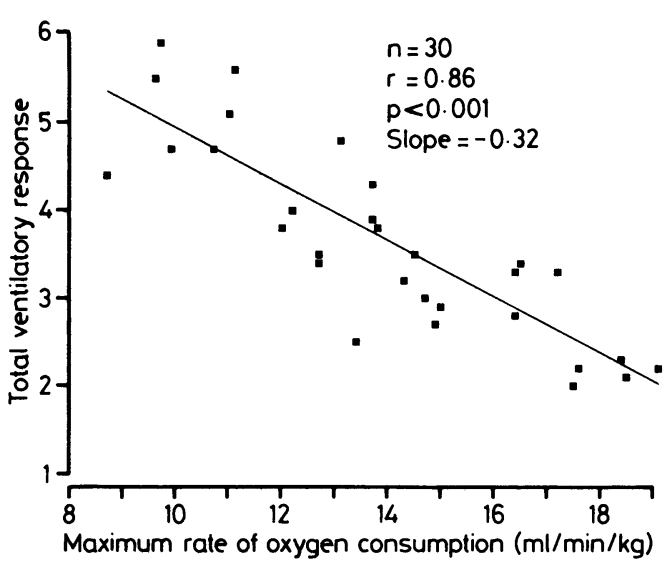

Figure 2 Relation between the total ventilatory response to exercise and the severity of chronic heart failure (measured as $\mathrm{VO}_{2}$ max) for all 30 patients.

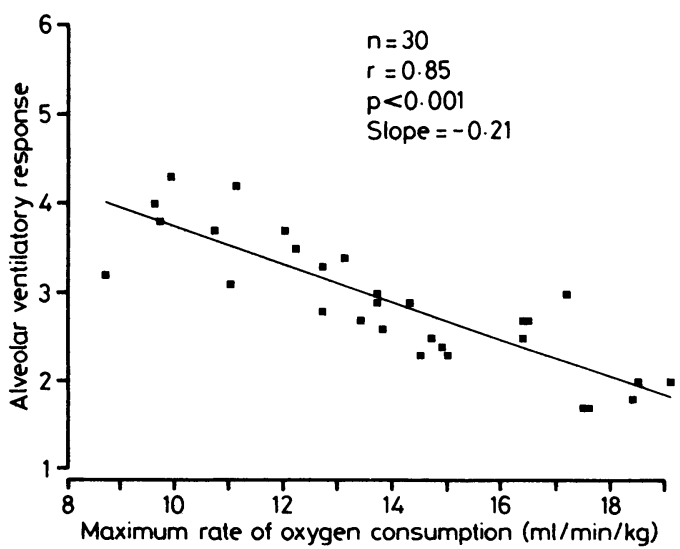

Figure 3 Relation between the alveolar ventilatory response to exercise and the severity of chronic heart failure (measured as $\mathrm{VO}_{2}$ max) for all 30 patients. 


\section{Discussion}

Our results show that in patients with heart failure the relation between total minute ventilation and the rate of carbon dioxide production is almost linear (fig 1). The slope of this relation is inversely related to the peak oxygen consumption on exercise (fig 2 ). There is a similar but less steep relation between peak oxygen consumption and the slope of the relation between alveolar ventilation and carbon dioxide production (fig 3).

The measurement of $\dot{\mathrm{VO}}_{2}$ max is now well established as an objective and reproducible index of the limitation of exercise capacity in patients with chronic heart failure and is a measure of the severity of heart failure. ${ }^{10}$ Thus the ventilatory response to exercise is also an indicator of the severity of heart failure. The only previous study to show such a correlation in individual patients was that of Fink et al. ${ }^{4}$ They studied bicycle exercise and found an inverse correlation $(\mathrm{r}=-0.59, \mathrm{p}<0.01)$ between $\mathrm{Vo}_{2}$ max and a derived value of minute ventilation at a rate of carbon dioxide production of $1 \mathrm{l} / \mathrm{min}$. The higher correlation coefficient in the present study $(r=-0.86$, $\mathrm{p}<0.001)$ may reflect chance variation or, alternatively, may result from the use of treadmill rather than bicycle exercise. Bicycle exercise is associated with lower values for $\mathrm{VO}_{2}$ $\max ^{12}$ and a frequent limiting symptom of fatigue rather than breathlessness. ${ }^{4}$ Indeed, Wilson et al in a more recent study conclude that "bicycle exercise cannot be used to measure $\mathrm{Vo}_{2}$ max". ${ }^{12}$

With regard to the mechanisms of exercise hyperpnoea in patients with chronic heart failure, our results show that correction for ventilation of anatomical dead space only reduced the gradient of the relation between ventilatory response and $\dot{\mathrm{VO}}_{2} \max$ by approximately one third. In consequence, we conclude that an altered respiratory pattern in patients with severe chronic heart failure only accounts for approximately one third of the increased exercise hyperpnoea seen in patients with heart failure (compare, for example, ventilatory response at a maximum rate of oxygen consumption of $10 \mathrm{ml} / \mathrm{min} / \mathrm{kg}$ in figs 2 and 3). Arterial blood gas tensions were not measured, but, assuming these remained constant, ${ }^{5-7}$ the results must also imply that approximately two thirds of the exercise hyperpnoea is due to an increased physiological dead space, and consequently a significant ventilation/perfusion mismatch. Our results further indicate that the magnitude of this abnormality is directly related to the severity of chronic heart failure as assessed by peak oxygen consumption on exercise. ${ }^{10}$

As in earlier studies ${ }^{34}$ we found a close linear correlation between minute ventilation and the rate of carbon dioxide production during exercise in patients with chronic heart.failure. The demonstration that the slope of this relation in individual patients was closely related to the severity of chronic heart failure suggests the possibility that this measurement may provide a simple and objective measure of severity of chronic heart failure. This measurement can be obtained without the patient being required to perform maximal exercise. A consequence is that obtaining the measurement is less unpleasant to the patient and not dependent on encouragement of the patient in the last few moments of an exercise test. The measurement is ideally suited to repeated evaluation.

1 Harrison TR, Pilcher C. Studies in congestive heart failure. II. The respiratory exchange during and after exercise J Clin Invest 1930;8:291-304.

2 Gazetopoulos N, Davies H, Oliver C, Deuchar D. Ventilation and haemodynamics in heart disease. Br Heart 1966;28:1-15.

3 Weber KT, Kinasewitz GT, Janicki JS, Fishman AP. Oxygen utilisation and ventilation during exercise in patients with chronic cardiac failure. Circulation patients with chr.

4 Fink LI, Wilson JR, Ferraro N. Exercise ventilation and pulmonary artery wedge pressure in chronic stable congestive heart failure. Am J Cardiol 1986;57:249-53.

5 Wilson JR, Ferraro N. Exercise intolerance in patients with chronic left heart failure: relation to oxygen transport and ventilatory abnormalities. Am J Cardiol 1983;51:1358-63.

6 Franciosa JA, Leddy CL, Wilen M, Schwartz DE. Relation between haemodynamic ventilatory responses in determining exercise capacity in severe congestive heart failure. Am J Cardiol 1984;53:127-34.

7 Rubin SA, Brown HV, Swan HJC. Arterial oxygenation and arterial oxygen transport in chronic myocardial failure at rest, during exercise and after hydralazine treatment. Circulation 1982;66:143-8.

8 Rubin SA, Nemerovski M, Brown HV, Siemienczuk D, Swann HJC. Mechanisms of dyspnoea on exertion in severe, chronic heart failure [Abstract]. Clin Res 1981;29:237A.

9 Davies N, Denison DM. Measurement of metabolic gas exchange and minute volume by mass spectrometry alone. Resp Physiol 1979;36:261-7.

10 Lipkin DP, Perrins J, Poole-Wilson PA. Respiratory gas exchange in the assessment of patients with impaired ventricular function. Br Heart $J$ 1985;54:321-8

11 Cotes JE. Lung function. 4th ed. Oxford: Blackwell Scientific Publications, 1979:244.

12 Wilson JR, Fink LI, Ferraro N, Dunkman WB, Jones RA Use of maximal bicycle exercise testing with respirator gas analysis to assess exercise performance in patients with congestive heart failure secondary to coronary artery disease or idiopathic dilated cardiomyopathy. $A m$ Cardiol 1986;58:601-6. 